\title{
CAUTION PROFILE AND DRIVING RECORD OF UNDERGRADUATE MALES*
}

\author{
Donald C. Pelz \\ Institute for Social Research. The University of Michigan, Ann Arbor, Mich.. U.S.A. \\ and \\ EDWARD KRUPAT \\ Department of Psychology. Boston College. Chestnut Hill, Mass., U.S.A. \\ (Receiced 3 January 1973: in revised form 12 Scptember 1973)
}

\begin{abstract}
BACKGROUND
Research on behavioral factors in automobile driving (e.g. perception, motivation, personality) is hindered by the fact that the ultimate criteria of driving ability-accidents or traffic violations-are relatively infrequent. The influence of any given behavioral factor is obscured, since a crash or citation depends on the confluence of several determinants which may converge only a few times in the course of a year.
\end{abstract}

In studying the effects of a particular training program, for example, one ordinarily has to use large samples and wait several months for the evidence to accumulate. The latter procedure is self-defeating if the effect of the program is real but temporary, since the likelihood of detection will diminish as the data-gathering period extends.

There is therefore a pressing need for intermediate criteria-measurement tools which can detect short-term changes in intervening variables which govern the likelihood of the occurrence of accidents or violations. The search for valid intermediate criteria has been frustrating. Verbal tests of knowledge. for example, seem to have little relationship-or even a negative relationship-with driving record [note. for example, Crancer et al.. 1971]. Performance tests such as simple reaction time have likewise been disappointing [Goldstein. 1961].

An alternative approach which appears somewhat more promising has been to measure the perceptual accuracy of drivers. Spicer [1964] devised a film showing eleven 34-min segments representing a variety of traffic situations: after each segment, subjects selected from a check-list whatever features they had noticed that were of importance to them. Drivers aged 15-17 with accidents were less accurate in perceiving essential features than were accident-free drivers.

Recent research by Burg [1971] has investigated visual acuity with a moving target. In a comprehensive study of 13,000 drivers with $3-y$ r driving records and nearly 8000 drivers

\footnotetext{
* Development of the Apprehension Meter was supported by the Highway Safety Research Institute. University of Michigan. from a grant by the Automohile Manufacturers Association (now Motor Vehicle Manufacturers Association]. Additional analysis has been conducted under grant MH 21276 from the National Institute of Mental Health. The apparatus was designed and built by Enest B. Therkelson. Films were used through courtesy of Allstate Insurance Company. Construction of derived measures was programmed by Ray E. Faith. The authors are indebted to Roberta S. MeConochic for help in preparing a preliminary analysis (Pelz. Krupat and McConochie, 19711.
} 
with 6-yr records. Burg examined numbers of accidents and of convictions as a function of sex, age, mileage and several vision measures. Among the latter. dynamic visual acuity - the ability to perceive details of an object when there is relative motion between the observer and the object-demonstrated "the strongest and most consistent relationships with driving record", although the correlations were still rather low.

The present research represents an attempt to design an intermediate criterion of driving record derived from perception. where the visual stimulus is dynamic rather than static, the subjects' response is continuous rather than episodic, and the criterion reflects not accuracy or knowledge but rather perceptual style, including dimensions such as promptness in detecting danger and non-abruptness of response. The research has parallels with several other lines of investigation. Using a model car simulation with accident repeaters and non-repeaters matched on age and mileage. Currie [1969] measured subject's speed in spotting potential collisions (measured by quickness in braking). as well as simple reaction time. While the two groups did not differ in reaction time, the accident non-repeaters perceived the danger sooner.

Perception and response with moving stimuli in a laboratory were studied by Hakkinen [1958], using an elaborate set of 14 performance tests yielding about 300 scores on $44 \mathrm{Hel}-$ sinki tram drivers and 52 bus drivers classified by accident experience over 8 yr of employment. In a Driving Apparatus Test the subject turned a wheel so as to keep a pointer on a "road" on a moving belt. while simultaneously responding by hand and foot levers to erratic signals. In an Expectancy Reaction Test the subject watched a kind of moving highway map, and was told to respond whenever two highways actually joined but not when they nearly joined or simply bridged one another; errors were prompted by a light which sometimes came on at actual joinings and sometimes at wrong points.

When a factor analysis was applied to 28 variables (including the accident criterion, a driver merit rating, and 26 scores selected from among the more valid and reliable ones in several tests), the highest accident loading appeared on a factor which Hakkinen called "attention", determined primarily by correct responses and absence of errors on the two tests above, indicating "correct motor responding within a specified time to a suddenly occurring signal". The next highest accident loading was on a factor of "involuntary control of motor functions", with poor control indicative of "hastiness, susceptibility to disturbances and motor restlessness". As in prior investigations the author found that simple reaction time did not distinguish safe from accident groups. but may indicale both "hastiness and the shifting of decisions to the motor level alone". These two factors are relevant to findings in the present study on respectively (a) prompt detection of danger and (b) nonabruptness of response to it.

A different approach on judgment of traffic hazards is the work of Adams [1968] who used a "stimulus accretion" technique with static targets. In the latest version of his apparatus the subject was given a colored photograph of a traffic situation seen from the driver"s seat, with the picture cut into squares. Starting from a pre-set square, $S$ removed one at a time under metronome pacing, to reveal a picture of the same situation 4 sec later after some hazard had appeared. The subject was instructed to stop whenever he "recognized the traffic situation" in the second picture and to describe what was important to him as a driver.

Adams" original assumption was that "a more competent. experienced driver would have a more efficient pattern of search for hazards and would therefore uncover and discern the hazard with the least number of blocks". In fact, the opposite was found. A lower 
Accident Index score-frequency of accidents over the past $10 \mathrm{yr}$, weighted by culpability and severity, in relation to exposure (mainly total miles driven)-was associated with more blocks removed before $S$ stopped, but in contrast with Spicer's finding there was no consistent correlation with number of hazards correctly identified. The author interprets these results as indicating the importance of a "caution vs impetuousness" dimension in accident occurrence, rather than a "knowledge" dimension.

Still another methodology is illustrated in a project carried out by the State of Washington Department of Motor Vehicles [Crancer et al., 1971], which utilized a driving simulator in which each subject watched three $23-\mathrm{min}$ films of varied driving environments. A series of 16 variables was scored, based on the subject's use of accelerator, brake, signals, steering and speed in relation to responses considered appropriate to specific situations. All but one of these was significantly related to good driving record, but 11 of the 15 variables were correlated negatively. The poor-record drivers had faster reaction time, and made fewer errors in steering, speed and turn signals; these skills together with superior knowledge of right-of-way laws (on a separate knowledge test) showed them to be "technically competent and confident". But they made more situation-missed errors; apparently their attention to steering, etc. impaired their ability to spot danger, indicating the importance of perception.

The good-record driver, by contrast, showed a bias toward caution; he tended to control by the accelerator rather than by the brake. The poor-record driver tended to approach at a higher speed, necessitating earlier application of the brake. "Thus the poor-record driver tended to be much more abrupt or jerky than the good driver in terms of vehicle control."

The results of this series of studies suggest that style of perceptual response will be more predictive of driving record than will accuracy as such or simple reaction time. The present procedure is designed to measure several dimensions of perceptual style which have been suggested by previous research such as overall level of caution, promptness of perception and abruptness of reaction.

\section{PROCEDURES}

With the apparatus used in this study, each subject watches a film as seen from the driver's eye view, and manipulates a handle to register the level of safety or danger he perceives from moment to moment. The film-projected with Cinemascope wide-angle lenswas adapted from films used in the Allstate-Link driving simulator, with all instructor's comments eliminated. The subject does not steer or brake as in the simulator.

The viewer's handle. which connects with a control box at the side of the chair, governs a needle on a meter face which is positioned in about the same location as a speedometer, and is dimly lit to be visible in the darkened projection room. The scale on the meter ranges from SAFE at one end, to UNSAFE at the other, with about 10 unmarked calibrations between. Subjects were instructed:

Your job is simply to report how safe or unsafe you feel as the driver at all times... On your right hand side there is a lever. By moving this lever you can move the pointer on the meter in front of you...If you felt completely at ease-if you were at rest or parked and could completely take your mind off driving-you would keep the meter at the SAFE endpoint. But, if you felt extremely threatened, very unsafe, or in immediate danger of being involved in a serious accident or mishap. you would move the meter all the way to the UNSAFE endpoint. And, of course. you can move the meter anywhere along the scale to best express your feelings. 
In this report, a high recording on the scale from Safe to Unsafe will be termed a high level of caution. Each viewer's caution level (or level of estimated danger) was recorded continuously by a stylus on a Brush strip-chart recorder moving at $1 \mathrm{~cm} / \mathrm{sec}$. The range of the meter was adjusted to cover an $8 \mathrm{~cm}$ wide scale which was scored from 0 (completely safe) to 100 (highly dangerous).

Although the apparatus was designed to let several viewers watch the same film. each responding on his individual meter, in the present study each subject was treated individually and was seated close enough to the screen (about $8 \mathrm{ft}$ ) to enhance the sensation of being on the highway. All subjects entered wholeheartedly into the task. Many remarked on its realism and their sensations of threat.

Each subject viewed $10 \mathrm{~min}$ of driving film, the first $5 \mathrm{~min}$ comprising a treatment segment-which is not of concern for the present analysis [see Krupat. 1971] - and the second 5 min comprising a measurement segment which supplied the data described below.

All subjects saw the same measurement segment of $5 \min (300 \mathrm{sec})$ of moderate city traffic, containing 10 mild hazards. This segment was not in a continuous sequence but was edited so as to provide the desired number and type of hazards. Subjects were instructed to expect some discontinuity, and they appeared able to disregard this.

When each viewer's caution record was examined, the point at which he noticed each hazard was identified - the place where his caution level changed from horizontal to rising: this was called his "point -of onset". Likewise, when the hazard had passed, it was possible to identify its "point of offset"- the spot at which the downward slant became again horizontal.

Among the 10 hazards in the measurement segment, we subsequently found that six were recognized by most subjects. with clear points of onset and of offset. The remaining four hazards, however, were ambiguous; some viewers failed to recognize either their appearance or their termination. The absence of a clear point of onset prevented the scoring of certain measures described below; hence these four events were omitted.

\section{Subjects}

Subjects were 60 undergraduate males, all licensed drivers drawn from the introductory psychology pool at the University of Michigan. Median age was 19; all but three were aged 18-21, with the oldest 25 . Median miles driven in the past year was 3400 .

In a questionnaire completed at the end of the experimental situation, more than half (36) indicated that they had had neither an accident nor a traffic citation (moving violation) during the preceding year. These were called the Safe Record group for the following analysis. The remainder were divided into a set of 13 with one or more crashes during the past year but no violations (Accidents only group), and 11 with violations only or both violations and accidents (Violations-or-both group, of whom two had both). Hence 25 per cent reported an accident and 18 per cent a violation.

In another study using a representative cross-section sample of young male drivers aged 16-24 in a suburban area adjacent to Detroit [Pelz and Schuman, 1971], about 23 per

* The absence of such a recognition point might be considered a perception failure; a corresponding measure of accuracy could be derived. For a reliable measure of accuracy, however. a different type of film would be needed-one with many hazards ranging from faint to distinct. The procedure we adopted in the film and the methods of analysis assumes that all viewers $d o$ recognize the hazards. and it measures various aspects of how' they respond. 
cent reported one or more accidents in the previous 1 -yr period and 24 per cent one or more violations, with an average annual mileage of about 11,000 . Hence our undergraduate sample drove one-third as many miles as the representative sample, but had about as many accidents and two-thirds as many tickets.

\section{Scores obtained}

From each viewer's 5-min recording of caution level during the measurement segment, scored from 0 to 100 on the paper belt, a series of scores was coded manually and several summary scores derived.

(A) Baseline. Each viewer's caution record was coded at $1-\mathrm{sec}$ intervals during the uneventful period between hazards - i.e. in that period of time between his "point of offset" for one hazard and his "point of onset" of the next (definitions of these points were given above), and also during the interval prior to the first hazard and after the last one. (Where an individual failed to show a clear point of onset or of offset for a single hazard. the average point for the total group was substituted.) For each viewer, his a verage across all such resting periods constituted his baseline.

(B) Relative time of onset. For each hazard the time of each individual's point of onset was expressed as a deviation from the total group's a verage time on onset. For each viewer the mean of these values was obtained for the six clearly-recognized hazards. All scores were calculated from a standard event on the film. Since the recording device was activated manually, it is possible that there are inaccuracies of up to $1 \mathrm{sec}$ as a result of this. A mechanical activation device would be preferable to assure accurate timing.

(C) Slope of onset. As described earlier, the point of onset for each hazard was marked when the caution record started to rise; a second mark was made when the record stopped rising. A straight line was drawn between these points and its angle from the horizontal measured in degrees. Computed for each viewer was the average slope of onset across six hazards.

(D) Maximum level. By inspection, the highest caution level for each hazard was coded; the viewer"s average of these across six hazards was computed.

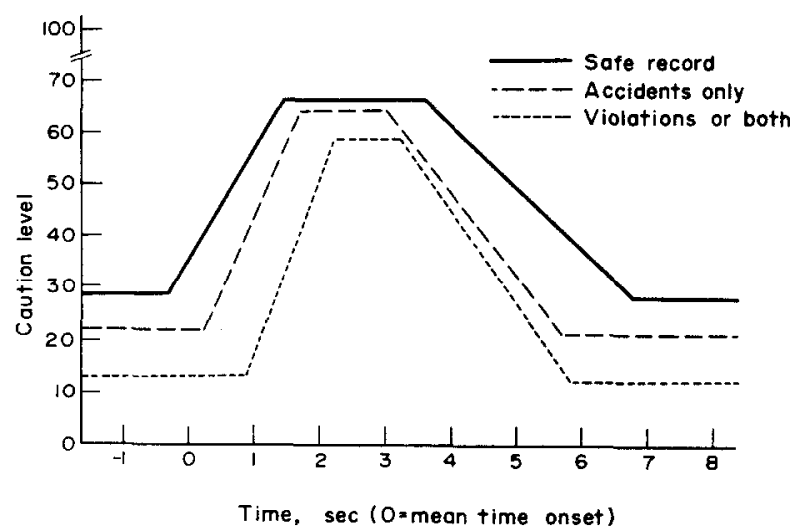

Fig. 1. Composite caution profile based on means in Table 1. On the time scale, zero is the point at which the average viewer began to respond. 
(E) Slope of offset. For each viewer a mark was made for each hazard when the caution record started downward; the point of offset (return to horizontal) was marked as described earlier. The angle of straight line between these points was recorded. and the viewer's a verage across six hazards was computed.

(F) Relative time of offset. As with measure (B), the time of offset for each hazard was expressed as a deviation from the total group's average time of offset for that hazard; for each viewer an average was obtained of these relative times across six hazards.

(G) Duration of hazard. The time elapsed between points of onset and offset respectively for each hazard was expressed in seconds, and the a verage duration across six hazards was computed.

If any individual lacked a point of onset or point of offset for one hazard. that incident was dropped in computing all of his summary scores (B) $(\mathrm{G})$. But if he lacked data on two or more hazards the individual was dropped from analysis. resulting in the loss of four cases. Final $N$ thus $=56$.

\section{Differences by driting record}

\section{RESULTS}

When the three driving record groups were compared on the various summary scores with one-way analysis of variance. statistically reliable differences appeared on five measures, as shown below in Table 1. Contrasts between the groups can be grasped more readily in terms of a composite caution profile as plotted in Fig. 1.

Table 1. Summary scores (across six hazards) for three driving record groups

\begin{tabular}{|c|c|c|c|c|c|c|}
\hline & \multicolumn{2}{|c|}{$\begin{array}{l}\text { A. Baseline } \\
\text { (caution scale) }\end{array}$} & \multicolumn{2}{|c|}{$\begin{array}{l}\text { B. Time of onset* } \\
\text { (in seconds) }\end{array}$} & \multicolumn{2}{|c|}{$\begin{array}{l}\text { C. Slope of onset } \\
\text { (in degrees) }\end{array}$} \\
\hline & Mean & S.D. & Mean & S.D. & Mean & S.D. \\
\hline Safe record & $28 \cdot 9$ & $18 \cdot 5$ & -0.3 & $1 \cdot 2$ & $60 \cdot 3$ & $7 \cdot 1$ \\
\hline Accidents only & $22 \cdot 2$ & 15.7 & 02 & 08 & 66.9 & $7 \cdot 6$ \\
\hline Violations or both & $12 \cdot 7$ & $18 \cdot 5$ & 09 & 09 & $69 \cdot 6$ & $11 \cdot 6$ \\
\hline Eta (correlation ratio) & 0.33 & & $0 \cdot 38$ & & 0.44 & \\
\hline$F$ & 3.57 & & $4 \cdot 43$ & & $6 \cdot 34$ & \\
\hline \multirow[t]{3}{*}{ Statistical significance } & 0.05 & & 0.05 & & 0.01 & \\
\hline & \multicolumn{2}{|c|}{$\begin{array}{l}\text { D. Maximum level } \\
\text { (caution scale) }\end{array}$} & \multicolumn{2}{|c|}{$\begin{array}{l}\text { E. Slope of offiset } \\
\text { (in degrees) }\end{array}$} & \multicolumn{2}{|c|}{$\begin{array}{l}\text { F. Time of olfset* } \\
\text { (in seconds) }\end{array}$} \\
\hline & Main & S.D. & Mean & S.D. & Mean & S.D. \\
\hline Safe record & $67 \cdot 2$ & $17 \cdot 2$ & $42 \cdot 9$ & $15 \cdot 5$ & 0.5 & 20 \\
\hline Accidents only & 663 & 19.7 & 520 & $10 \cdot 8$ & $-0 \cdot 6$ & $1 \cdot 4$ \\
\hline Violations or both & 585 & 240 & 547 & 158 & -04 & 13 \\
\hline Eta (correlation ratio) & 0.17 & & $0 \cdot 34$ & & $0 \cdot 26$ & \\
\hline$F$ & 0.83 & & $3 \cdot 36$ & & 1.95 & \\
\hline Statistical significance & & & $0 \cdot 05$ & & $\cdots$ & \\
\hline
\end{tabular}

\begin{tabular}{|c|c|c|}
\hline & $\begin{array}{l}\text { G. D } \\
\operatorname{lin} s\end{array}$ & \\
\hline & Mein & S.D. \\
\hline Safe record & $7 \cdot 0$ & 1.6 \\
\hline Accidents only & 55 & 1.7 \\
\hline Violations or both & 4.9 & 20 \\
\hline Eta (correlation ratio) & 0.49 & \\
\hline$F$ & 7.27 & \\
\hline Statistical significance & 0.01 & \\
\hline
\end{tabular}

\footnotetext{
* Time of onset and of offet for each hazard is expressed as a deviation from the overall mean for that hazard.
} 
The first feature to be noted is that the three driving groups differed not only in their response to hazards but in their baseline caution during the uneventful intervening periods (part A of Table 1). The Safe Record group appeared to remain on their guard even when the situation seemed non-dangerous. whereas the Violations-only group were most relaxed with a baseline caution level only half as high.

When the hazard appeared. the Safe Record group responded to it soonest-the point of onset was 1.2 sec earlier than the Violations-only group (Table 1B). Although the differences were not large they were sufficiently precise (note small standard deviations) to be statistically significant.

After a hazard was recognized. the Violations group responded to it abruptly-the slope of onset measured in degrees was steepest (Table 1C); the Safe Record group had the most gradual onset slope. with the Accidents group intermediate. The differences in slope were not large but they were significant.

In terms of maximum caution level, differences among the driving record groups were non-significant (Table 1D). although the Safe Record group tended to be more cautious at the height of danger than did the Violations-only group.

When the danger passed. the slope of offset (Table IE) behaved in the same way as the slope of onset. The Violations group dropped most sharply, the Accidents group a little more slowly. and the Safe Record group declined gradually. In short, the style of responding to a hazard both at its beginning and at its end was most controlled for the Safe Record group, and most abrupt for the Violations group.

The time of offset (Table 1F) was not significantly different among the three groups. although the Safe Record group tended to be slower in returning to their normal baseline.

Finally the reader will note (Table $1 \mathrm{G}$ ) that in terms of total duration of each hazard-the period of time over which the viewer remained cautious - the Safe Record group had the longest duration and the Violations group the shortest.

It may be useful at this point to draw a composite portrait of the response style of each driving record group. The Safe Record drivers retained the highest level of caution during the baseline period between hazards. They were alert to danger, as shown by an early response to each hazard. Their level of caution then rose in a gradual or controlled fashion. and declined gradually: and they remained on guard the longest. They appeared consistently alert and controlled.

The Accidents-only group were intermediate in the baseline level of caution. and somewhat slow in responding to a hazard. When they did notice the danger they responded rather sharply, but then they relaxed rather quickly. In short, the Accidents group appeared somewhat carcless.

The liolations-or-thoth drivers were most relaxed during uneventful periods but responded sharply when a hazard appeared. Even at the height of danger they were less threatened than the other groups. and when the danger passed they quickly dropped their guard. Their typical posture, in short, was casual and abrupt.

\section{Measurement reliability consistency across hazards}

The measurement reliability of the summary scores were estimated from the consistency in responses through time. For scores $(\mathrm{B})-(\mathrm{G})$, covariances among the component scores for six hazards were used to compute Cronbach's alpha [Cronback. 1951; Bohrnstedt. 1969] with results as shown in Table 2. For score A, 10 component scores were availablethe baseline intervals preceding each of 10 incidents. 
The baseline measure was found to be highly consistent. with $\varkappa=0 \cdot 99$. as were slope of offset and maximum level (alpha's of $0.88,0.90$ respectively). Time of offset and duration were moderately high (alpha's of 0.71). Hence, baseline. slope of offset and duration can be called both valid (differentiated by driving record) and reliable.

Table 2. Reliability coefficients (Cronbach's alphilf for summary scores and caution index. based on intercorrelations among scores for six hazards

\begin{tabular}{ll}
\hline & Alpha \\
\hline A Baseline & 0.99 \\
B Time of onset & $0 \cdot 48$ \\
C Slope of onset & 0.55 \\
D Maximum level & $0 \cdot 90$ \\
E Slope of offset & $0 \cdot 88$ \\
F Time of offset & $0 \cdot 71$ \\
G Duration & 0.71 \\
Caution index, based on & \\
$\quad$ mini-indexes for six hazards & 0.85 \\
\hline
\end{tabular}

The final two scores-time of onset and slope of onset-had only modest reliabilities of 0.48 and 0.55 respectively, yet both of them were valid with respect to driving record. In fact the latter had a correlation ratio with driving record almost as high as the extremely reliable baseline. It is encouraging to note that these two scores plus duration related significantly to driving record despite modest reliability. Reliability could of course be improved simply by lengthening the 5 -min measurement film and incorporating more hazards. (The last entry in Table 2 will be explained later).

Intercorrelations among the seven summary scores

Since seven scores in Table 1 were all derived from a single, continuous caution recording, it is important to consider the statistical independence of each of the measures from the others. These correlations are given in Table 3.

Table 3. Correlations among seven summary scores*

\begin{tabular}{|c|c|c|c|c|c|c|}
\hline \multicolumn{7}{|c|}{ A Baseline } \\
\hline $0 \cdot 27$ & \multicolumn{6}{|c|}{ B (Early) point of onset } \\
\hline 0.33 & $0 \cdot 25$ & \multicolumn{5}{|c|}{ C (Gradual) slope of onset } \\
\hline 0.70 & 0.29 & $-0 \cdot 16$ & \multicolumn{4}{|c|}{ D Maximum level } \\
\hline 0.33 & $-0 \cdot 15$ & 0.60 & -0.22 & \multicolumn{3}{|c|}{ E (Gradual) slope of offset } \\
\hline $0 \cdot 36$ & $-0 \cdot 28$ & 0.45 & 0.17 & 0.65 & F Lat & oint of offset \\
\hline 0.52 & $0 \cdot 34$ & 0.59 & 0.35 & 0.56 & 0.81 & G Duration \\
\hline
\end{tabular}

* Direction of scores has been reversed on measures $B$. C. and $E$ so that a high score as re-labeled characterizes Safe Record drivers.

To facilitate study, the direction of measures B. C, and E has been reversed so that a high value on all variables now characterizes the Safe Record drivers. The reader will note that almost all correlations were positive among the five scores which related significantly to driving record in Table 1 : baseline, early point of onset, gradual slope of onset, gradual slope of offset and duration. 
The objection may be raised that the scoring procedure makes some of the measures necessarily interdependent. Duration of a hazard is expected to be longer if slope of both onset and offset is gradual; hence one is not surprised at the respective correlations $r_{C G}=$ 0.59 and $r_{\mathrm{EG}}=0.56$. Also, since duration is simply the difference between times of onset and offset respectively, it readily follows that $r_{\mathrm{FG}}=0.81$. However, $r_{\mathrm{BG}}=0.34$ is surprisingly modest. Other relationships, also. were not as high as the scoring procedure would suggest.

On the other hand. several correlations appeared which were by no means necessary. A high baseline does not require a long duration, yet $r_{\mathrm{AG}}=0.52$. A viewer who rises steeply when a hazard appears need not relax immediately when it disappears, yet $r_{\mathrm{CE}}=$ 0.60 . In such instances the consistency appears to be psychological rather than artifactual.

In short: on the "caution profile" scores in Tables 1 and 3, viewers generally seemed to respond in a consistent fashion that was compatible with driving record. Yet the several scores were somewhat independent. There did not appear to be a single dimension of "cautious vs relaxed". but a multi-faceted style of response.

\section{Caution index}

The significant relationships for several of the profile scores in Table 1, and the moderate consistency among them in Table 3. are promising. They suggest that it may be useful to construct a composite "caution index" and to find out how well such an index can predict the driving record of each viewer.

To guard against inflation introduced by a statistical fitting process, given the limited number of cases in the present study, the following relatively crude procedure was therefore used: (a) for the five measures showing a significant relationship with driving recordbase-line level. time of onset. slope of onset. slope of offset and duration-standardized scores were obtained in terms of means and standard deviation; (b) signs were reversed on the middle three. as indicated in Table 3 ; (c) the resulting scores were summed with equal weight to obtain an index for each individual.

The distribution of this caution index for each of the driving record groups is displayed in Fig. 2. Two main features may be noted. First: the index differentiated fairly well



Fig. 2. Distribution of caution index for three driving record groups. 
between Safe Record drivers and the other two groups. Among the former the large majority (79 per cent) scored above zero: among the latter the large majority (72 per cent for the two groups combined) scored below zero. To put it another way: in the total sample of 56. if one predicted that all viewers with a positive index had a safe record. and all with a negative index had an accident or violation. one's prediction would be correct for over three-quarters ( 77 per cent) of the cases.

Second: the caution index differentiated hardly at all. however. between the Accidents and Violations group.

Another way to look at the degree of differentiation is shown in Table 4. The mean scores of the three groups on the caution index differed in a consistent fashion. and the

Table 4. Differentiation of three driving record groups on caution index*

\begin{tabular}{lccc}
\hline & $(N)$ & Meall & S.D. \\
\hline Safe record & $(34)$ & $1 \cdot 42$ & $2 \cdot 60$ \\
Accidents only & $(12)$ & $-1 \cdot 44$ & 2.87 \\
Violations or both & $(10)$ & $-3 \cdot 27$ & +.09 \\
Eta & & 0.55 & \\
Eta-squared = variance & & 0.31 & \\
accounted for & & $11 \cdot 64$ & \\
Statistical significance & & 0.001 & \\
\hline
\end{tabular}

* Caution index $=$ sum of $=$-scores for: baseline. (earty) time of onset, (gradual) slope of onset, (gradual) slope of offset and duration.

differentiation was statistically significant (as would be expected. since the components of the index each differentiated significantly among the driving groups). The mignitude of relationship $(\eta=0.55)$ was substantially higher than for any of the components in Table 1 , suggesting that the components were not overlapping but measured different dimensions. The proportion of variance accounted for $\left(\eta^{2}=31\right.$ per cent) indicates a promising degree of predictability. but with room for improvement.

One is curious about the 21 per cent of Safe Record drivers with negative index scores. These are indistinguishable from drivers with infractions. One possible interpretation. which would be testable by a follow-up on these subjects, is that their response habits are far from safe-making them candidates for a crash or a ticket in the coming year.

\section{Measurenent reliability of caunion index}

To estimate measurement reliability of the caution index as a whole. we examined its consistency through time. First. for each of the six hazards. a "mini-caution index" wals constructed from the five profile scores for that hazard. in a manner parallel to that for the overall caution index. Standardized scores were formed for each hazard in terms of respective means and standard deviations. After signs of three items were reversed as indicated earlier, the =-scores were added to produce a mini-caution index for each viewer on that hazard.

Covariances among the mini-indexes for the six hazards were used to construct Cronbach's alpha which, as shown in the last entry in Table 2 above, had a reasonably high value of 0.85 . To raise this figure it would be simple to employ a longer stimulus film with (say) twice as many incidents. 


\section{SUMMARY AND DISCUSSION}

In the procedure described above. each of 60 undergraduate males watched a 5 -min film of highway driving as seen from the driver"s seat and recorded a moment-by-moment judgment of danger by means of an "Apprehension Meter". Three groups divided on driving record-Safe Record, Accidents only and Violations-or-both-were found to differ signifcantly on five scores comprising a "caution profile". It will be meaningful to summarize the results by linking them with several investigations cited earlier in which good-record drivers appeared more prompt in detecting but more deliberate in responding. compared to poor-record drivers. The Safe Record group recognized danger sooner and longer. and they responded to this information in a more controlled manner.

Each of the measures which constitute our apprehension profile can be linked with one or more characteristics in previous studies. Thus Currie [1969] in a model-car simulation found that low-accident drivers perceived danger sooner. In Häkkinen`s [1958] elaborate set of performance tests, he identified an accident-related attention factor which depended on correct perception and prompt reaction to an erratic series of sudden signals. Our procedure did not attempt to measure accuracy as such but was able to detect how promptly. the viewer noticed the hazard, by the point at which his apprehension level began to rise. Safe Record drivers were most prompt and Violations drivers were slowest.

Crancer and associates [1971]. using a driving simulator, found that poor-record drivers had superior manipulative skills and knowledge, but tended to be more abrupt or jerky than good record drivers. Similarly, Häkkinen [1958] obtained a second accident-linked factor of "involuntary motor control" characterized by hasty and distractable responses. A parallel result in our apprehension data was that the Safe Record group were more controlled. They showed the most gradual slope of both onset (apprehension increase) and offset (apprehension decline). whereas the Violations group showed the steepest slopesthat is, they were more abrupt.

Adams [1968] measured how much information the viewer required to recognize the hazard in a series of highway photographs. He found -contrary to initial expectationsthat drivers with a low accident record took longer before they were willing to stop searching, and he postulated a dimension of caution vs impetuousness. Correspondingly, our Safe Record group had the highest haseline level of caution between hazards, and longest duration of elevated caution (interval between points of onset and offset for each hazard). The baseline caution score was among the most valid and clearly was the most reliable of the five scores. Under non-stressful conditions, Safe Record drivers remained most alert while Violations drivers were most casual.

The five differentiating scores were combined into a multidimensional "caution index" with a reliability (alpha coefficient) of 0.85 . This was able to classify correctly 77 per cent of the cases as either safe or non-safe. although it did not differentiate the latter as to accidents or violations.

\section{Physiological measures and driving record}

In the original experiment which provided data for the present analysis, Krupat [1971] measured maximum caution level and emotional arousal by electrical skin resistanceboth long-term basal resistance levels (BRL) as well as the more familiar galvanic skin response (GSR) noted by momentary deflections in skin resistance. He found parallels between the conscious judgment of danger obtained with the Apprehension Meter and the physiological skin-resistance measures. Maximum caution level averaged over the six 
hazards correlated $0 \cdot 35$ with BRL (inverted), and 0.24 with GSR. indicating mild positive relationships between perception and arousal.

Additional existing evidence indicates that rates of GSR rise in proportion to density and difficulty of traffic events [Hulbert. 1957: Michaels, 1960]. Taylor [1964] measured GSR in relation to different road conditions during actual driving. He found that the GSR rate per minute remained relatively constant. but that on road sections with higher actual risk the GSR experience per mile was higher; its spatial distribution followed the accident distribution. Taylor reasoned that the driver must speed up on safe stretches and slow down on dangerous ones, in order to maintain a constant GSR rate with respect to time. He proposed that: "driving is a self-paced task governed by the level of emotional tension or anxiety which the driver wishes to tolerate".

Taylor did not attempt to link GSR level with driving record. and we have found no studies which demonstrate a clear relationship between GSR and driving record.* An indirect study is one by Preston [1969] who did not use the individual's driving record but rather his insurance classification based on occupation and age. on the premise that insurance premiums are roughly proportional to accident experience. Preston found little difference between high and low risk categories on GSR rates in town driving. but significantly higher GSRs for the high-risk category on country driving (i.e. their ratio of town to country GSR was lower). She suggested that "in town traffic most of the GSRs are generated by the actions of other drivers, whereas on the open road the GSRs are self-generated by the subjects' driving".

An analysis was made of BRL in relation to driving record in the present study during the baseline periods and during each of the hazards. but no significant relationships were found. This lack of relationship can possibly be understood in terms of the Taylor and Preston studies which suggest that accident and safe groups might differ not in overall GSR activity, but rather in their differential response to certain driving situations (town vs country roads, or risky vs non-risky road segments). One can hypothesize that in selfpaced driving where the degree of risk is under the driver's control, accident drivers might be found to tolerate (or to seek) higher GSR rates than safe drivers. On the other hand. in externally paced driving where degree of risk is controlled by traffic conditions, the difference in GSR rates might be nil or opposite.

In the present study, of course. the "driving task" is externally paced by the film. Hence it is not surprising that no relationships were found between basal resistance level (BSL) and driving record, during each of 10 baseline points and six hazards.

\section{TOPICS FOR FUTURE RESEARCH AND APPLICATION}

These results offer promise for use of the caution profile as an intermediate criterion of safe driving, and as a predictor of the ultimate criteria of driving crashes and violations. Before such applications are attempted. however, additional steps would be needed. such as: (a) repetition on a larger and more heterogenous sample of drivers; (b) mechanical coordination of projector and recording. and electronic recording of responses; (c) experimentation to test susceptibility of the scores to "faking"; and (d) experimentation to test the impact of manipulable inputs such as supervised road experience, fatigue, and alcohol.

\footnotetext{
* Häkkinen [1958. pp. 65-66] reports a 1939 study by Cobb on 3600 drivers which found zero correlation between an accident index and GSR, and a 1930 study by Weiss and Lauer which observed a near-significant tendency for longer duration of GSR (departure of resistance from average value) in an accident vs a control group.
} 
In addition, further research will be needed to determine whether different types of training will be found to have a differential short-term effect on the cautious scores-an effect which later shows up in long-term driving record.

If so, the Apprehension Meter may be able to diagnose poor habits of perception. and to provide a means of evaluating training programs aimed at improving cognitive and perceptual abilities.

The apparatus was also designed not only for the purpose of measurement (diagnosis or evaluation) but also for training as such. It was designed to be used with groups of up to 20 viewers at one time watching the same film. Each viewer's meter is constructed so as to activate six color-coded zones. The color-coded signals from each viewer's meter are fed into an instructor's console by means of which he can monitor the moment-by-moment responses of all viewers. The console permits the instructor to freeze (stop and hold) both the film and the collective set of responses at any moment when he feels it would be instructive.

At this point, the instructor may activate a display panel-a larger version of his console-to display publicly the frozen responses of all viewers by means of colored lights corresponding to the zones on each meter face. The display panel will indicate how each viewer's sense of danger compares with that of the other members. The instructor can then stimulate a discussion on what each viewer perceived at that moment, and why the judgments of driving danger differed.

In preliminary trials with this feature, driver education students found it challenging to compare their responses with each other, making public what is ordinarily a private process of seeing and judging. They could test their alertness moment-by-moment against that of fellow watchers. Some considered this experience more instructive than a traditional safety film, since they could participate actively instead of watching passively.

\section{REFERENCES}

Adams. J. F. [1968] Measurement of hazard judgment by a stimulus accretion technique. Safety Research and Education Project. Teachers College. Columbia University, New York City. February 1968.

Bohrnstedt. G. W. [1969] A quick method of determining reliability and validity of multiple-item scales. Am. Sociol. Rer. 34, (4). 542-548.

Burg. A. [1971] Vision and Driving: a report on research. Human Factors, 13, (1). 79-87.

Crancer. A. Jr.. with the assistance of Wallace. J.. Delay. J.. Paulsrude. S. and Rodell. M. [1971] Proiect METER (Machine Examination Teaching. Etaluation and Re-education). State of Washington Department of Motor Vehicles, Office of Research and Technology. January 1971.

Cronbach. L. J. [1951] Coefticient alpha and the internal structure of tests. Psychometrika 16, $289-321$.

Currie, L. [1969] The perception of danger in a simulated driving task. Ergonomics 12, 841-849.

Goldstein. L. G. [1961] Research on human variables in safe motor vehicle operation: a correlational summary of predictor variables and criterion measures. George Washington University. Driver Behavior Research Project. Washington. D.C.

Häkinen. S. [1958] Traffic Accidents and Dricer Characteristics. Finland's Institute of Technology. Scientific Researches No. 13, Helsinki.

IIulbert. S. F. [1957] Drivers' GSRs in traffic. Percep. Motor Skills, 7, 305-315.

Krupat. E. [1971] Perceived threat as a function of prior experience. Doctoral dissertation. University of Michigan. Ann Arbor. Mich.

Michaels. R. M. [1960] Tension responses of drivers generated on urban streets. Highway Res. Board Bull. 271, $29-44$.

Pelz. D. C.. Krupat. E. and McConochie, R. S. [1971] Apprehension level and driving record of undergraduate males (a preliminary analysis). Survey Research Center, University of Michigan. Ann Arbor. Mich.. July 1971.

Pelz. D. C. and Schuman, S. H. [1971] Are young drivers really more dangerous after controlling for exposure and experience? J. Safety Res. 3, (2), 68. 79. 
Preston. B. [1969] Insurance classifications and drivers' galvanic skin response. Ergonomics 12, (3). 437-446. Spicer. R. A. [1964] Human factors in traffic accidents. Final report. USPHS Research Grant No. AC-00055. May 1964. Department of Health. Honolulu. Hawaii.

Taylor. D. H. [1964] Drivers' galvanic skin response and the risk of accident. Ergonomics 7, 439-451.

\begin{abstract}
With a device called an Apprehension Meter. 60 undergraduate males watched a 5 -min film of highway driving as seen from the driver's seat and recorded a moment-by-moment judgment of degree of danger. or caution level. From responses to six discrete hazards and to intervening uneventful periods. several summary scores were derived of which five differentiated significantly by driving record. The Safe Record group remained more cautious during the uneventful (baseline) periods. reacted to hazards sooner but more gradually, relaxed more gradually after their disappearance, and consequently remained alert to each hazard longer. The Violations group were at the other extreme. and the Accidents group intcrmediate. Hence the Safe group appeared more prompt in detecting danger, but less abrupt in responding to it. than did the groups with infractions. In terms of consistency across six hazards or 10 baselines. reliability (Cronbach's alpha) ranged from 0.48 to 0.99 . A composite caution index had an alpha of 0.85 across hazards and correctly classified 77 per cent of subjects as either safe or non-safe. The results were found consistent with those in other studies.
\end{abstract}

